\title{
Texture evolution and Swift effect in NiAl
}

\author{
Rainer Glüge*, Thomas Böhlke, and Albrecht Bertram \\ Otto-von-Guericke-Universität Magdeburg, Fakultät für Maschinenbau, Institut für Mechanik, \\ Universitätsplatz 2, D-39106 Magdeburg, Germany
}

\begin{abstract}
Skrotzki et al. [4] performed free end torsion tests on polycrystaline $\mathrm{NiAl}$ at 1000 to $1300^{\circ} \mathrm{K}$, where the texture evolution and the Swift effect have been measured. A material model is formulated and implemented to simulate the torsion tests and compared our results to the experimental findings.
\end{abstract}

(c) 2006 WILEY-VCH Verlag GmbH \& Co. KGaA, Weinheim

\section{Introduction}

$\mathrm{NiAl}$ is an intermetallic compound with a high melting point (about $1660^{\circ} \mathrm{C}$ ), good corrosion resistance, and good electrical and thermal conductivity at a low density compared to conventional high temperature alloys, but suffers from brittleness below $500^{\circ} \mathrm{C}$. In order to overcome this brittleness, a fine grained microstructure is desirable, which can be achieved by extensive deformation [5]. This leads to a strong texture evolution. Therefore it is impotrant to understand the texture evolution in NiAl. An elastic viscoplastic polycrystal model based on the Taylor assumption is implemented into the commercial finite element software ABAQUS. Three different simulation techniques have been used, namely a deformation driven process for a material point, an FE model of the torsion test, and a simple shear deformation of an RVE. The RVE consists of 15625 elements, the microstructure is modeled with 350 periodic Voronoi cells. Periodic boundary conditions have been applied. The RVE simulations have been performed in order to estimate the applicability of the Taylor model.

\section{The material model}

The material model uses the plastic transformation $\mathbf{P}$ that evolves through

$$
\mathbf{P}^{-1} \dot{\mathbf{P}}=-\sum_{\alpha=1}^{N} \dot{\gamma}_{\alpha} \tilde{\mathbf{M}}_{\alpha}, \dot{\gamma}_{\alpha}=\dot{\gamma}_{0} \operatorname{sgn}\left(\tau_{\alpha}\right)\left|\frac{\tau_{\alpha}}{\tau_{\alpha}^{C}}\right|^{m}, \tilde{\mathbf{M}}_{\alpha}=\tilde{\mathbf{d}}_{\alpha} \otimes \tilde{\mathbf{n}}^{\alpha}, \tau_{\alpha}=\mathbf{T}_{e}^{\prime} \cdot \tilde{\mathbf{M}}_{\alpha}
$$

where $\tilde{\mathbf{d}}_{\alpha}$ is the slip direction and $\tilde{\mathbf{n}}^{\alpha}$ is the slip plane normal of the crystallographic slip system (see $[3,7])$. The elastic law is given by

$$
\mathbf{T}_{e}^{2 P K}=\tilde{\mathbb{C}}\left[\frac{1}{2}\left(\mathbf{C}_{e}-\mathbf{I}\right)\right], \quad \mathbf{C}_{e}=\mathbf{F}_{e}^{T} \mathbf{F}_{e}, \quad \mathbf{T}_{e}^{\prime}=\mathbf{C}_{e} \mathbf{T}_{e}^{2 P K},
$$

where $\mathbf{F}_{e}=\mathbf{F P}$. The isotropic hardening is modeled by

$$
\tau^{C}=\tau_{0}^{C}+\left(\tau_{\infty}^{C}-\tau_{0}^{C}\right)\left(1-\exp \left(\frac{-\theta_{0} \gamma}{\tau_{\infty}^{C}-\tau_{0}^{C}}\right)\right)+\theta_{\infty} \gamma, \quad \gamma=\int \sum_{\alpha=1}^{N}\left|\dot{\gamma}_{\alpha}\right| \mathrm{d} t .
$$

The tilde indicates a formulation with respect to the intermediate configuration. The simulations have been carried out with the following material parameters, calibrated at $723^{\circ} \mathrm{C}: C_{1111}=188 \mathrm{GPa}, C_{1122}=$

\footnotetext{
* e-mail: rainer.gluege@mb.uni-magdeburg.de, Phone: +00 4939167 11329, Fax: +00 493916712863
} 
$123 \mathrm{GPa}, C_{1112}=93 \mathrm{GPa}, \dot{\gamma}_{0}=10^{-3}, m=13, \tau_{0}^{C}=12 \mathrm{MPa}, \tau_{0}^{C}=27 \mathrm{MPa}, \theta_{0}=120 \mathrm{MPa}, \theta_{\infty}=$ 12.5MPa. The elastic constants are taken from [1], the hardening parameters are adapted to compression tests found in [2], from where also the flow rule parameters have been taken. The active slip systems are the primary $\langle 100\rangle\{110\}$ systems and the secondary $\langle 110\rangle\{110\}$ systems above $500^{\circ} \mathrm{C}$. The temperaturedependent ratio $k=\tau_{2}^{C} / \tau_{1}^{C}\left(k=2\right.$ at $700^{\circ} \mathrm{C}$, [2]) is assumed to be unaffected by the hardening. The numerical time integration of the internal variables has been carried out using the backward Euler scheme.

\section{Experiments vs. simulations, texture evolution and Swift effect}

The experiments [4] have been performed with two textured samples, one with an initial $\langle 100\rangle$ fiber and the other with a $\langle 111\rangle$ fiber parallel to the torsion axis. In case of the $\langle 100\rangle$ sample monotonic shortening occurs, while the $\langle 111\rangle$ sample initially lengthens before monotonic shortening occurs. This general behaviour could be reproduced in the FE simulations for moderate strains. A variation of $k$ from 5 at $T \leq 500^{\circ} \mathrm{C}$ to 1 at $T \geq 900^{\circ} \mathrm{C}$ shows a weakening of the Swift effect. This contradicts the experimental findings [6]. The asymptotic shear texture can be reached by rotating the $\langle 100\rangle$ fiber $90^{\circ}$ around the transverse direction, and a superimposed rotation of the orientations within the fiber towards the component which finally has a $\langle 110\rangle$ direction parallel to the torsion axis. In case of the $\langle 100\rangle$ sample the texture predictions using Taylor's assumption suit well to the experimentally measured texture, and are slightly sharper than the texture predictions from the RVE simulations. The texture evolution accords to the explanation given above. For the $\langle 111\rangle$ sample the simulations using Taylor's assumption overestimate a texture component which is formed temporarily before reaching the proper asymptotic shear texture, while another texture component is underestimated. The results form the RVE calculations suit better to the experimental findings.

\section{Conclusions}

Taylor's assumption can be applied for the texture prediction in NiAl. For large deformations the predictions are in good agreement to experimental results, when there is time to establish an asymptotic texture, but texture components may be over- or underestimatet in intermediate states. RVE calculations yield better results. The Swift effect could be reproduced up to moderate strains. The magnitude of the Swift effect is found to be temperature-dependent in the experiments, and is more pronounced at elevated temperatures. This temperature-dependence enters through various mechanisms, like the ratio $k$ of the secondary to the primary critical resolved shear stress, hardening behaviour, and recrystallization. $k$ approaches 1 at high temperatures, which leads to a more isotropic behaviour and therefore weakens the Swift effect, as it was found in the simulations. A variation of the hardening parameters did not show any noteworthy influence on the Swift effect. Therefore it seems that recrystallization has a strong influence on the Swift effect, and should be included into a material model for simulations at high temperatures.

\section{References}

[1] D. B. Miracle, Acta Met. et Mat.41/3, 649-684 (1992)

[2] J. Fischer-Bühner: Mechanismen der Mikrostruktur- und Texturentwicklung von polykristallinem NiAl. (Shaker Verlag, Aachen, 1998)

[3] T. Böhlke: Crystallographic Texture Evolution and Elastic Anisotropy: Simulation, Modeling, and Applications. (Shaker Verlag, Aachen, 2001)

[4] W. Skrotzki et al., Mat. Sci. Eng. A329-331, 235-240 (2002)

[5] W. Skrotzki et al., Text. Microstruct. 35(3/4),163-173 (2003)

[6] W. Skrotzki, B. Klöden, private communication

[7] A. Bertram: Elasticity and Plasticity of Large Deformations - an Introduction. (Springer Verlag, BerlinHeidelberg, 2005) 